\title{
Black holes and Boyle's law - the thermodynamics of the cosmological constant
}

\author{
Brian P. Dolan * \\ Department of Mathematics, Heriot-Watt University \\ Colin Maclaurin Building, Riccarton, Edinburgh, EH14 4AS, U.K. \\ Maxwell Institute for Mathematical Sciences, Edinburgh, U.K.
}

August 19, 2014

\begin{abstract}
When the cosmological constant, $\Lambda$, is interpreted as a thermodynamic variable in the study of black hole thermodynamics a very rich structure emerges. It is natural to interpret $\Lambda$ as a pressure and define the thermodynamically conjugate variable to be the thermodynamic volume of the black hole (which need not bear any relation to the geometric volume). Recent progress in this new direction for black hole thermodynamics is reviewed.
\end{abstract}

PACS nos: 04.60.-m; 04.70.Dy

\section{Introduction}

If asymptotically flat space-time contains a black hole then a certain volume of space is hidden from an observer at infinity by the event horizon. The volume of space available is less because the black hole excludes a volume that is inaccessible to the observer. It is however not immediately obvious how to define the volume of a black hole in any geometric sense: for example in the line element for a Schwarzschild black hole, described by Schwarzschild co-ordinates $(t, r, \theta, \phi)$, the "radial" co-ordinate $r$ is time-like inside the event horizon at $r=r_{h}$, so integrating the areas of constant $t$ shells of radius $r$ from $r=0$ to $r=r_{h}$ seems nonsensical from the point of view of the space-time geometry of a black hole, because $r$ represents a time for $r<r_{h}$. For black holes in the presence of a cosmological constant $\Lambda$ there is the added ingredient of the vacuum energy: if

*e-mail: B.P.Dolan@hw.ac.uk 
a volume of space is hidden from an observe at infinity, is the corresponding vacuum energy also hidden from them?

To avoid problems with a cosmological horizon, consider for the moment the case of asymptotically anti-de Sitter space-time (AdS). Then there is a negative vacuum energy density $\epsilon=\Lambda<0$. If a volume could be defined and a black hole with a volume $V$ were present then a negative energy $\epsilon V$ would be excluded from the point of view of an observer at infinity. Does this mean that positive energy is available to them?

Suppose we could define a volume for a black hole in some meaningful way, then the total energy $U$ of the black hole would be not just its mass (as defined by an asymptotic observer at infinity, the ADM mass [1-3] or its equivalent in asymptotically AdS space-time [4) but the mass plus the vacuum energy hidden behind the event horizon,

$$
U=M+\epsilon V .
$$

Since the equation of state for a cosmological constant, $\Lambda=-8 \pi G_{N} P$, is that the pressure $P=-\epsilon$, we have

$$
M=U+P V .
$$

In the thermodynamics of black holes the mass is usually interpreted as the thermal energy and the cosmological constant is taken to be fixed. The first law, in its simplest form, is then

$$
d M=T d S,
$$

where $T$ is the Hawking temperature and $S$ the Bekenstein-Hawking entropy. But if the internal energy is $U$, then $M$ in equation (2) is the enthalpy $H(S, P)$ of the thermodynamic system, the Legendre transform of $U(S, V)$,

$$
M=H(S, P)=U(S, V)+P V .
$$

This interpretation of the black mass as enthalpy in a thermodynamic sense was first suggested in [5], based on scaling arguments and the Smarr relation [6].

The idea that the cosmological constant might be considered as a variable has been suggested many times over the years, the earliest example seems to be [4, 7]-9] and it has re-surfaced many times [10-[16].

When the cosmological constant is fixed, we still have (3), but if the cosmological constant is varied infinitesimally this becomes

$$
d M=T d S+V d P,
$$

where, by definition,

$$
V=\left.\frac{\partial H}{\partial P}\right|_{S} .
$$

This gives a thermodynamic definition of the black hole volume [5]. Note that this has no a priori relation to any notion of geometric volume, it is a purely 
thermodynamic concept, we shall therefore define $V$ in (5) to be the thermodynamic volume [17. As already mentioned the definition of a geometric volume of a black hole is not straightforward, but there is a number of suggestions in the literature, [18-21] one of which [19] does actually co-incide with the thermodynamic volume in asymptotically flat-space times, but not otherwise.

The idea of varying the cosmological constant is sometimes criticised on the basis that $\Lambda$ dictates the asymptotic form of the metric and it does not make sense to compare space-times with different asymptotic behaviour. But opening our minds and embracing the thermodynamic potential as a real physical entity in itself, putting aside questions about the underlying space-time which

produced it, gives a new perspective on the physics. That is the inference of the chain of ideas presented here: the thermodynamic potential is obtained by integrating out the underlying space-time and, having done so, we are asked to forget where the potential came from and just ask what physics it gives us. With this attitude in mind we should be careful about how phrases such as "varying the cosmological constant" are interpreted, we do not mean that $\Lambda$ becomes a function of time but rather we wish to ask the question, "what would the physics look like if $\Lambda$ were different?"

In this review recent progress in the understanding of thermodynamic volumes will be described. It is traditional to highlight the similarities between black holes and ordinary thermodynamic systems, but there are also some very important differences and $\$ 2$ gives a quick summary of the four laws of black hole thermodynamics with a discussion of some of the aspects that differ from more usual systems. 33 describes work to date on including $\Lambda$ as a thermodynamic variable in 4-dimensional black holes, 4 covers the 3 -dimensional case of asymptotically AdS BTZ black holes and $\$ 5$ describes the rich structure of higher dimensional black holes, including triple points and re-entrant phase transitions. $\sqrt{6}$ is devoted to the cosmological constant in the boundary field theory of the AdS/CFT correspondence and gauge/gravity duality.

\section{The four laws of black hole thermodynamics}

The laws of black hole thermodynamics are usually stated as follows (see e.g. [22]).

(0) The surface gravity $\kappa$ is constant on the event horizon.

With the Hawking temperature [23]- 225], $T=\frac{\hbar \kappa}{2 \pi}$, this associates a unique temperature to the black hole in a way that agrees with our intuitive feeling for thermodynamic systems, that the temperature is the same everywhere in the system.

(1) For a black hole of mass $M$, angular momentum $J$ and electric charge $Q$,

$$
d M=T d S+\Omega d J+\Phi d Q
$$


where the entropy $S=\frac{1}{4} \frac{A}{\hbar G_{N}}$ is proportional to the area of the event horizon 23]- 27.

This identifies the internal energy, $U(S, J, Q)$, with the mass and $T=\frac{\partial U}{\partial S}$ but, as argued in the introduction, this is only consistent for asymptotically flat black holes. When there is a non-zero cosmological constant this should be modified to

$$
d M=T d S+V d P+\Omega d J+\Phi d Q,
$$

where $M$ is the enthalpy. Of course the first law is usually written in terms of the thermal energy

$$
U=M-P V
$$

and (7) is equivalent to [5, 17, 28,

$$
d U=T d S-P d V+\Omega d J+\Phi d Q
$$

which reduces to the usual expression for black holes 22] when $P=0$. An earlier version of (10) was proposed in 29] for spherically symmetric spacetimes, but with $U$ replaced with $M$. A $P d V$ term in the first law was also considered in the context of cosmological horizons and inflation, with no black hole, in [30], and in teleparallelism theories in [31]-33].

(2) The area of the event horizon never decreases (in any classical process).

This relates to the identification of the entropy as being proportional to the area. Of course it is well known that Hawking radiation can result in the area of the event horizon decreasing, but when the entropy of the emitted radiation is included in the balance the total entropy of the Universe does not decrease.

(3) It is not possible to achieve $\kappa=0$ in a finite number of steps.

While the above identifications are very nice and resonate intuitively with thermodynamic principles it is also important to appreciate the differences between the thermodynamics of black holes and more standard systems. Some of these are:

1. In an ordinary system the temperature is not only a property of the system as a whole, it is also a property of parts of the system. A macroscopic volume of gas at a given temperature can be divided into two parts, each half the original volume, and the parts will have the same temperature as the original (this is the intensive nature of temperature). This is not true for black holes, a black hole cannot in general be broken up into two pieces each with the same temperature as the original. 
2. In a thermodynamic system in $d$ space dimensions all extensive variables scale the same way. If lengths change by a factor $\lambda$ then the volume changes as $V \rightarrow \lambda^{d} V$, the entropy as $S \rightarrow \lambda^{d} S$, the particle number (or charge) as $N \rightarrow \lambda^{d} N$, and the internal energy $U(S, V, N)$ as $U \rightarrow \lambda^{d} U$. Scaling arguments then imply 34] that the Gibbs free energy

$$
G(T, P, N)=U-T S+P V=N \mu
$$

is equal to $N \mu$ where $\mu$ is the chemical potential. Since, by definition,

$$
d G=-S d T+V d P+\mu d N
$$

equation (11) gives the Gibbs-Duhem relation

$$
\mu d N=-S d T+V d P .
$$

A complete Legendre transform to intensive variables trivially gives zero,

$$
\Xi(T, P, \mu):=G-N \mu=0 .
$$

There are even axiomatic approaches to thermodynamic theory that elevate homogeneous scaling to the status of an axiom [35].

For black holes the scaling of the thermodynamical variables (using Newton's constant to convert length into mass and $c=1$ ) is

\begin{tabular}{|l|c|}
\hline Thermodynamic Variable & Dimension \\
\hline Mass, $M$ & $D-3$ \\
Entropy, $S$ (area) & $D-2$ \\
Angular momenta, $J^{i}$ & $D-2$ \\
Volume, $V$ & $D-1$ \\
Electric Charge, $Q$ & $D-3$ \\
Temperature, $T$ & -1 \\
Angular velocity, $\Omega_{i}$ & -1 \\
Pressure, $P(\Lambda)$ & -2 \\
Electric potential, $\Phi$ & 0 \\
\hline
\end{tabular}

Scaling then gives the Smarr relation [6],

$$
(D-3) M=(D-2) T S+(D-2) \boldsymbol{\Omega} . \mathbf{J}-2 P V+(D-3) \Phi Q
$$

(the notation $\boldsymbol{\Omega}$.J is used because there can be more than one angular momentum in five or more dimensions). In particular the Gibbs-Duhem relation in the infinitesimal form (13) has no analogue in black hole thermodynamics. It would be an interesting project to develop an axiomatic approach to black hole thermodynamics based on inhomogeneous scaling.

3. In black hole thermodynamics it is in fact crucial that the complete Legendre transform to intensive variables, $\Xi(T, P, \Omega, \Phi)$ is not zero. This is because $\Xi$ is related to the Euclidean action $I_{E}$ by 36

$$
I_{E}=\beta \Xi \text {. }
$$


In ordinary thermodynamics, for a system of $N$ non-interacting independent particles, the partition function factorises as $Z=z^{N}$, where $z$ is the partition function for a single particle. The analogue of this in black hole thermodynamics would presumably be some kind of multi-centre black hole soliton, a combination of $N$ single black hole solutions, such that the Euclidean action of the multi-centre solution is $N$ times the Euclidean action of a single black-hole, i.e. the Euclidean actions would need to be additive. But the non-linearities of General Relativity make this difficult to achieve. If a solitonic multi-centre black hole with non-vanishing additive action were to exist it would be the gravitational analogue of a free particle in ordinary thermodynamics.

4. The Hawking temperature of a Schwarzschild black hole is inversely proportional to the mass, $T=\frac{\hbar}{8 \pi G_{N} M}$, resulting in the famous negative heat capacity of black holes, a black hole in empty space will radiate and lose mass thus increasing its temperature making it radiate even more. This signals an instability that would normally make any such states unobservable. In ordinary thermodynamics a negative heat capacity would usually be considered to be unachievable in a real macroscopic system - in principle a fluctuation could take a system into such a state, but it would be so fleeting as to be unobservable. But for a solar mass black hole the Hawking temperature is of the order $10^{-8} \mathrm{~K}$ and the lifetime many orders of magnitude greater than the age of the Universe, hence such black holes are expected to persist as real objects for a long time.

It makes sense to consider negative heat capacity objects in black hole thermodynamics. While it is possible to stabilise them, e.g. by introducing a negative cosmological constant [37, it is not necessary to do so in order to discuss their thermodynamics.

5. The instability of asymptotically flat black holes is a persistent feature in any dimension. It was shown in 38 that all asymptotically flat rotating, electrically neutral, black holes are unstable and this was extended to include the charged case in [39]. The proof is a nice example of one of the differences between black hole thermodynamic and ordinary thermodynamic systems. Local thermodynamic stability is determined by the convexity properties of thermodynamic potentials, see e.g. Ref. 34. In the entropy representation complete stability requires that the entropy be a concave function of its arguments and, conversely, in the energy representation the internal energy should be a convex function of its arguments, which are all extensive. Thus, for a black hole including rotation and charge, we have $U(S, V, J, Q)$. Denoting the extensive variables by $X^{A}$ complete local thermodynamic stability requires that the Hessian

$$
U_{A B}=\frac{\partial U}{\partial X^{A} \partial X^{B}}
$$

be a positive definite matrix, in the sense that all its eigenvalues are positive. 
But it was shown in [39], using the Smarr relation (15), that there exists a negative norm vector of the bi-linear form $U_{A B}$ when $P V \leq 0$, regardless of the values of $J$ and $Q$, and this vector was explicitly constructed as a linear combination of the $X^{A}$. For fixed $J$ and $Q$ the norm of the vector becomes positive only ifl 1

$$
P V>2\left(\frac{D-2}{D-1}\right)(T S+\mathbf{\Omega . J})
$$

independent of the charge $Q$ (the co-efficient in (18) is determined by the scaling dimensions of the extensive variables). In ordinary thermodynamics the norm of the equivalent vector is always zero - homogeneous scaling of ordinary thermodynamics implies that there is always a direction of neutral stability - in black hole thermodynamics this is not the case.

6. The entropy of a solar mass black hole is essentially the area of the event horizon measured using Planck lengths. It is colossal, of the order of $10^{78}$. Using Boltzmann's formula (with $k_{B}=1$ ), $S=\ln (\mathcal{W})$, this implies that the available number of microstates (whatever those microstates are) 2 is $\mathcal{W} \approx e^{10^{78}}$.

An alternative statement of the third law, due to Nernst, is that the entropy should vanish at zero temperature. There is nothing pathological about zero temperature black holes: a rotating black hole has a lower Hawking temperature than a non-rotating one of the same mass,

$$
T=\frac{\hbar}{4 \pi M} \frac{\sqrt{M^{4}-J^{2}}}{\left(M^{2}+\sqrt{M^{4}-J^{2}}\right)},
$$

(with Newton's constant $G_{N}=1$ ) and the temperature vanishes for the maximum angular momentum $J=M^{2}$. Astrophysical black holes can have angular momenta close to the extremal value and an extremal Kerr black hole has an entropy that is half the entropy of a Schwarzschild black hole of the same mass. For an extremal solar mass black hole we again have an entropy of order $10^{78}$, for a zero temperature object! However there is no a priori contradiction with the Nernst statement of the third law here: the Nernst statement assumes that the ground state is non-degenerate, if the ground state is degenerate with degeneracy $g_{0}$, and there is a mass gap, then the entropy would be $S=\ln g_{0}$ at zero temperature. But it

\footnotetext{
${ }^{1}$ It is assumed that $\boldsymbol{\Omega} . \mathbf{J} \geq 0$. If it is not then the moment of inertia must have at least one negative eigenvalue, signalling another source of instability.

${ }^{2}$ While there are models of quantum gravity that allow the entropy to be calculated in terms of microstates [40, the focus of this review is on thermodynamics rather than statistical mechanics. Not because the latter is not important, it is even more important than thermodynamics when it is tractable, but because at the present time the microscopic theory underlying black hole thermodynamics, presumably a theory of quantum gravity, is still not well understood.
} 
would seem that compatibility with the third law implies that the ground state degeneracy of a solar mass black hole is huge. This already tells us something, usually a ground state degeneracy is lifted by perturbations unless a symmetry protects it - Nature may be telling us that some powerful symmetry implies large ground state degeneracies for quantum gravity that are protected against being lifted by perturbations.

\section{$3 \quad D=4$ space-time dimensions}

Having laid down the rules in the previous section, this section applies then to four dimensional space-times.

\subsection{Asymptotically anti-de Sitter black holes}

Consider first the simplest case of a neutral, non-rotating black hole. For asymptotically AdS Schwarzschild space-time, with and $\Lambda=-\frac{3}{L^{2}}$ interpreted as a pressure $P=-\frac{\Lambda}{8 \pi G_{N}}$, the line element is

$$
d s^{2}=-f(r) d t^{2}+\frac{1}{f(r)} d r^{2}+r^{2}\left(d \theta^{2}+\sin ^{2} \theta d \phi^{2}\right),
$$

with

$$
f(r)=\left(1-\frac{2 G_{N} M}{r}+\frac{r^{2}}{L^{2}}\right) .
$$

The thermodynamic volume in fact evaluates to the naïve Euclidean result for a sphere of radius $r_{h}, 5$

$$
V=\frac{4 \pi}{3} r_{h}^{3}
$$

This is no longer true for rotating black holes, the thermodynamic volume of the asymptotically AdS Kerr metric does not look like any geometric volume. The line element in this case, including an electric charge, is [4]

$d s^{2}=-\frac{\Delta}{\rho^{2}}\left(d t-\frac{a \sin ^{2} \theta}{X} d \phi\right)^{2}+\frac{\rho^{2}}{\Delta} d r^{2}+\frac{\rho^{2}}{\Delta_{\theta}} d \theta^{2}+\frac{\Delta_{\theta} \sin ^{2} \theta}{\rho^{2}}\left(a d t-\frac{r^{2}+a^{2}}{X} d \phi\right)^{2}$,

where

$$
\begin{aligned}
\Delta & =\frac{\left(r^{2}+a^{2}\right)\left(L^{2}+r^{2}\right)}{L^{2}}-2 m r+q^{2}, \quad \Delta_{\theta}=1-\frac{a^{2}}{L^{2}} \cos ^{2} \theta, \\
\rho^{2} & =r^{2}+a^{2} \cos ^{2} \theta, \quad X=1-\frac{a^{2}}{L^{2}} .
\end{aligned}
$$

The metric parameters $m$ and $q$ are related to the mass and charge by

$$
M=\frac{m}{X^{2}}, \quad Q=\frac{q}{X}
$$


and the angular momentum is $J=a M$.

The mass (and hence the enthalpy) can be expressed in thermodynamic variables 28

$$
M=H(S, P, J, Q)=\frac{1}{2} \sqrt{\frac{\left(S+\pi Q^{2}+\frac{8 P S^{2}}{3}\right)^{2}+4 \pi^{2}\left(1+\frac{8 P S}{3}\right) J^{2}}{\pi S}} .
$$

The thermodynamic volume evaluates to [42, 43]

$$
V=\left.\frac{\partial H}{\partial P}\right|_{S, J, Q}=\frac{2}{3 \pi H}\left[S\left(S+\pi Q^{2}+\frac{8 P S^{2}}{3}\right)+2 \pi^{2} J^{2}\right] .
$$

In terms of the geometric parameters $a, q$ and $L$ in the metric (23) this is

$$
V=\frac{2 \pi}{3}\left\{\frac{\left(r_{h}^{2}+a^{2}\right)\left(2 r_{h}^{2} L^{2}+a^{2} L^{2}-r_{h}^{2} a^{2}\right)+L^{2} q^{2} a^{2}}{L^{2} X^{2} r_{h}}\right\} \geq \frac{4 \pi}{3} r_{h}^{3} .
$$

A geometric definition of the volume of a black hole was given in [19] which in fact agrees with (28) for neutral black holes in the asymptotically flat limit, but they give different results when $L$ is finite (and so $\Lambda<0$ ).

The formula for the thermodynamic volume in terms of geometric variables highlights the fact that $V$ is equal to $\frac{4 \pi}{3} r_{h}^{3}$ if and only if $a=0$, i.e. for $J=0$ the volume reduces to the Euclidean result (22) for any $q$, they differ only for $J \neq 0$. Comparing this with the entropy

$$
S=\frac{\pi\left(r_{h}^{2}+a^{2}\right)}{X} \underset{a \rightarrow 0}{\longrightarrow} \pi r_{h}^{2}
$$

we see that the entropy and the volume are not independent when $J=0$, even for charged black holes. This can lead to subtleties in taking Legendre transforms [4].

The inequality in (28) is interesting, since rotating black holes necessarily deviate from spherical symmetry it implies that non-symmetric black holes have a smaller surface to volume ratio than spherically symmetric black holes of the same radius. This is contrary to our Euclidean intuition where the geometry with the smallest surface to volume ratio is a perfect sphere, any deviation from perfect symmetry increases the surface to volume ratio - the isoperimetric inequality of Euclidean geometry. This led the authors of Ref. 42 to postulate a reverse isoperimetric inequality for thermodynamic volumes of asymptotically AdS black holes: that the entropy inside a horizon of a given volume $V$ is maximised for Schwarzschild-AdS.

When discussing thermodynamic potentials it is important to bear in mind what the correct control parameters are [34. For example the thermal energy $U(S, V, J, Q)$ is a function of volume and

$$
P=-\left(\frac{\partial U}{\partial V}\right)_{S, J, Q} .
$$


To express $U$ as a function of $P$,

$$
U(S, P, J, Q)=U\left(S,-\frac{\partial U}{\partial V}, J, Q\right),
$$

implies a differential equation whose solution requires an undetermined constant. There is nothing wrong with writing $U(S, P, J, Q)$ as long as we remember that some information is lost in doing so. This is not necessary for the case in hand though as the Legendre transform (9) can be performed explicitly and the internal energy determined in terms of the natural thermodynamical variables 43

$$
\begin{array}{r}
U(S, V, J, Q)=\left(\frac{\pi}{S}\right)^{3}\left[\left(\frac{3 V}{4 \pi}\right)\left\{\left(\frac{S}{2 \pi}\right)\left(\frac{S}{\pi}+Q^{2}\right)+J^{2}\right\}\right. \\
\left.-|J|\left\{\left(\frac{3 V}{4 \pi}\right)^{2}-\left(\frac{S}{\pi}\right)^{3}\right\}^{\frac{1}{2}}\left(\frac{S Q^{2}}{\pi}+J^{2}\right)^{\frac{1}{2}}\right] .
\end{array}
$$

The presence of the $P d V$ term in (10) has physical consequences, it is possible to extract more energy in a Penrose process in asymptotically AdS space-time than in asymptotically flat space-time. The maximal efficiency for an electrically neutral black hole in the former case is [43] 51.8\%, while the value for the latter case is [22] $1-\frac{1}{\sqrt{2}}=29.3 \%$. The corresponding figures for a charged rotating black hole are $75 \%$ and $50 \%$ respectively.

As mentioned above when $J=0$ the thermodynamic volume (28) reduces to the Euclidean result $\frac{4 \pi}{3} r_{h}^{3}$, which is not independent of the entropy $S=\pi r_{H}^{2}$. It was observed in [44 that this has the amusing consequence that the adiabats for a Carnot cycle are also isochoric $(V=$ const) and hence are vertical lines in the $P-V$ plane. In terms of the familiar adiabatic condition $P V^{\gamma}=$ const, re-written as $P^{\frac{1}{\gamma}} V=$ const, this is equivalent to the statement that $\gamma=\infty$. In this sense non-rotating black holes behave like a gas of molecules with an infinite number of excitable internal degrees of freedom.

The introduction of the pressure as another thermodynamic variable in black hole physics expands our view of the thermodynamic picture. It was shown in Ref. 28, that an asymptotically AdS rotating black hole has a phase transition, when $J$ is held fixed, between large and small black holes. This was originally discussed in terms of fixed $\Lambda$ and can be seen by plotting curves of fixed $J$ in the $T-S$ plane. As $J$ is increased, keeping $\Lambda$ fixed, there is a critical value $J_{c}$ at which there is a second order phase transition, the transition is first order for $J<J_{c}$ and there is only one phase for $J>J_{c}$, while at $J=0$ the small black holes disappear. This phase transition, which we shall call the CKK phase transition, is distinct from the Hawking-Page phase transition [37. The latter occurs at constant angular velocity, $\Omega$, when the free energy of the black hole is larger than the free energy of AdS with no black hole, but with thermal radiation rotating with angular velocity $\Omega$, and the black hole decays to AdS 
plus radiation. Discussion of the Hawking-Page phase will be postponed to later in this section and we shall focus on the CKK phase transition first.

The CKK phase transition can also be pictured by fixing $J$ and varying $P$ (or $\Lambda$ ). When isotherms are plotted in the $P-V$ plane the similarity with the van der Waals gas becomes striking [43. While the equation of state $T(P, V)$ cannot be obtained in closed form for non-zero $J$ (that would require extracting the roots of an eighth order polynomial), a virial expansion can be performed and critical exponents extracted at the critical point. The critical exponents $\alpha$, $\beta, \gamma$ and $\delta$, are defined in the usual way (with reduced temperature $t=\frac{T-T_{c}}{T_{c}}$ and reduced volume $\left.v=\frac{V-V_{c}}{V_{c}}\right)$,

- the specific heat diverges as $C_{V} \sim|t|^{-\alpha}$;

- the jump in volume just below $T_{c}$, between the gaseous (large black hole) phase, $v_{>}$, and the liquid (small black hole) phase, $v_{<}$, vanishes as, $v_{>}-v_{<} \sim|t|^{\beta}$

- the isothermal compressibility diverges as $\kappa_{T} \propto|t|^{-\gamma}$;

- at the critical temperature the pressure $\left|P-P_{c}\right| \propto|v|^{\delta}$.

For the CKK phase transition the exponents are mean field

$$
\alpha=0, \quad \beta=\frac{1}{2}, \quad \gamma=1, \quad \delta=3,
$$

putting the asymptotically AdS Kerr black hole in the same universality class as the van der Waals gas. [46, 45. The same exponents are found in a singularity in the isothermal moment of inertia tensor [47.

This phase transition can be analysed in terms of the Gibbs free energy for the system, $G(T, P, J)=M-T S$. This is a multi-valued function of its arguments and the most stable phase corresponds to the lowest branch of $G$. For an asymptotically AdS, electrically neutral, rotating black hole $G$ is plotted in figure 1, as a function of $T$ for $J=1$ and various values of the pressures near the critical value $P_{c}$. The thermodynamically stable configuration is the one of lowest $G$ and the lower envelope of $G$ is a concave function. For pressures below the critical pressure there is a kink where $\left|\frac{\partial G}{\partial T}\right|_{P, J}$ is discontinuous, corresponding to a first order phase transition, for pressures above the critical value the kink disappears. The singularity associated with the critical point is that of a swallowtail in the language of catastrophe theory, 48, $A_{4}$ in the $A-D-E$ classification of singularities 49 .

When viewed in the $P-T$ plane the phase boundary of the CKK transition re-enforces the analogy with the van der Waals liquid-gas transition. There is a line of first-order phase transitions terminating in a critical point where there is a second order phase transition, as shown in figure 2

Electrically charged black holes show similar features, indeed the first suggestion of van der Waals type behaviour for a black hole was an analogy between the non-rotating charged black hole equation of state, $\Phi(Q, T)$ at fixed $P$, and the 


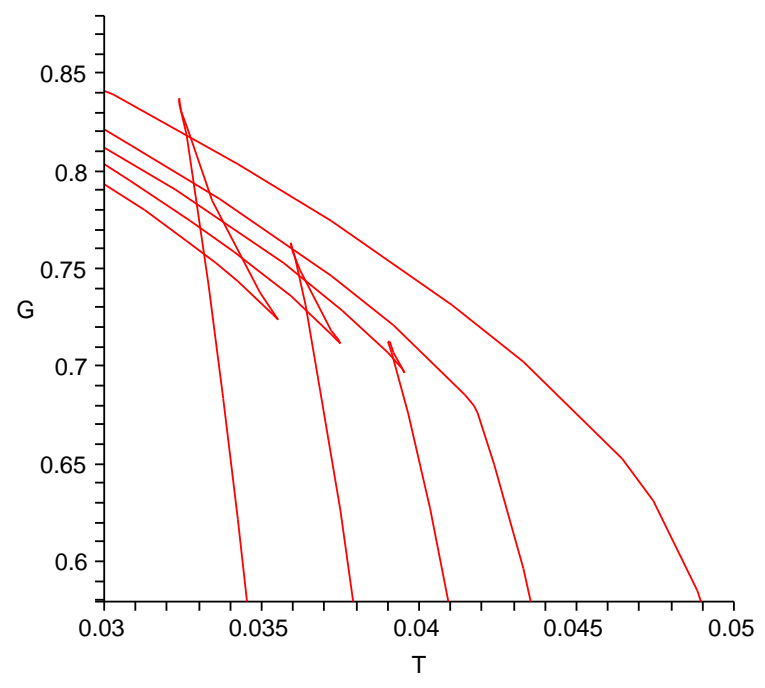

Figure 1: The Gibbs free energy in $D=4$, as a function of $T$ at various pressures and fixed $J=1$.

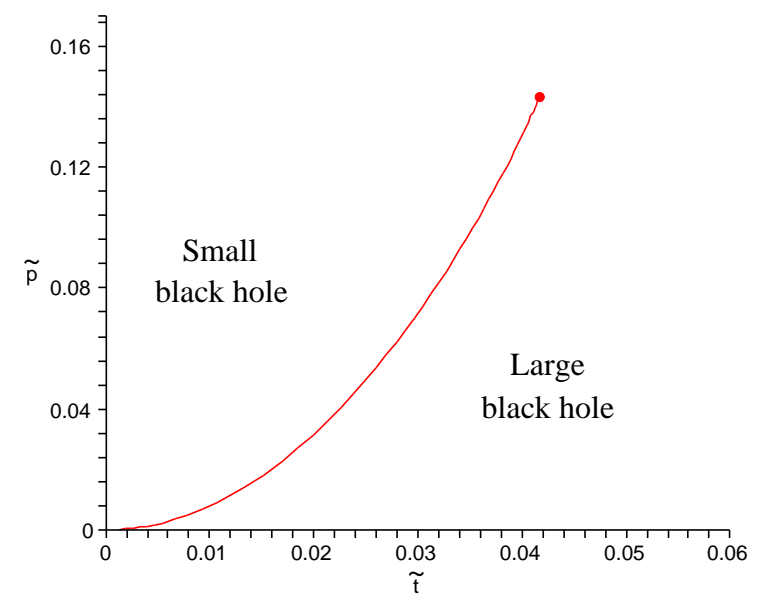

Figure 2: Co-existence curve of the CKK transition with fixed $J$, between large and small black holes, in the $P-T$ plane. There is a line of first order transitions terminating in a critical point which is a second order phase transition with mean field exponents 45, 46. The pressure and temperature are here rendered dimensionless using suitable powers of $J$, (with $\left.G_{N}=1\right) \tilde{t}=T \sqrt{J}$ and $\tilde{p}=$ $16 \pi P J$. 
van der Waals equation, $P(V, T)$, based purely on charge and electric potential with no reference to volume, [50, 51] an analogy which was pursued in [52]-55]. Bringing the thermodynamic volume into the picture makes this analogy even closer. In the non-rotating case the equation of state can be determined exactly

$$
P=\frac{T}{2}\left(\frac{4 \pi}{V}\right)^{\frac{1}{3}}+\frac{1}{8 \pi}\left(\frac{4 \pi}{V}\right)^{\frac{2}{3}}+\frac{Q^{2}}{8 \pi}\left(\frac{4 \pi}{V}\right)^{\frac{1}{3}}
$$

and the critical point has mean field exponents, which can be seen in both in the $Q-\Phi$ plane [55] and in the $P-V$ plane [56].

When both $J$ and $Q$ are non-zero there is a line of second order phase transitions in the $J-Q$ plane encircling the origin 28, each point of which has mean field critical exponents [46]. The $J=0$ case uniquely has the property that the thermodynamic volume equals the naïve Euclidean volume, and the volume is therefore not independent of the entropy, rotation is necessary to free the thermodynamic volume from the entropy. The critical point in the charged rotating case was studied in 57 .

Non-linear modifications of electrodynamics have also been considered in the context of varying $\Lambda$. For example one can replace the Maxwell action with the Born-Infeld action [58, 59]

$$
\mathcal{L}_{B I}=-b^{-4}\left(\sqrt{-\operatorname{det}\left(g+b^{2} F\right)}-\sqrt{-\operatorname{det} g}\right),
$$

(where $g_{\mu \nu}$ the space-time metric, $F_{\mu \nu}$ the Maxwell field strength and $b$ a fixed constant with dimensions of length). Charged asymptotically AdS black hole solutions of the Einstein-Born-Infeld system are known [60-62] and have been analysed from the perspective adopted here $(b$ is a new dimensionful parameter which affects the Smarr relation). Divergences in the heat capacity on the spinodal curve ${ }^{3}$ were examined in 63, 64 and exponents characterising the divergence were calculated in 65. At the critical point, where the two branches of the spinodal curve meet, the character of the divergence changes and the critical exponents associated with solutions of the Born-Infeld action are again mean field [45. A new feature in Born-Infeld electromagnetism is that there is a range of $b$ for which there can be a discontinuous jump in the value of the lowest branch of the Gibbs free energy - a zeroth order phase transition [45].

For charged non-rotating black-holes the van der Waals type behaviour first observed in [50, 51] was for fixed electric charge. If the system is coupled to a charge reservoir and the electric potential fixed there is no critical point. However if, in addition, the black hole is given a magnetic charge a rich phase space structure re-emerges [66] with large and small black hole phases and a second order phase transition. The inclusion of a magnetic charge on the black hole is an essential ingredient for understanding the quantum Hall effect from the AdS/CFT point of view [67, 68].

\footnotetext{
${ }^{3}$ The spinodal curve is defined to be the curve, in the $T-S$ or the $P-V$ plane, on which response functions, such as heat capacity or compressibility, diverge.
} 
Maxwell's equal area law can be used to identify the temperature of the phase transition, equating free energies in the two phases determines the temperature of the transition when it is below the critical temperature, and this was investigated in [47, 69, 70. This is however one of those situations where one must be aware of the differences between the thermodynamics of black holes and more familiar systems: in a fluid there is a definite ratio of the amount (molar mass) of fluid in the liquid phase to the amount in the gaseous phase at any point on the horizontal segment of the isotherm constructed using the equal area law, given by the "lever rule", 34 but there is only one black hole in the CKK transition and it does not seem sensible to apportion it into a partly large black hole and partly small black hole.

Knowing the equation of state allows one to calculate a compressibility for a rotating black hole, 71 either adiabatic

$$
\kappa_{S}=-\frac{1}{V}\left(\frac{\partial V}{\partial P}\right)_{S, J, Q}
$$

or isothermal

$$
\kappa_{T}=-\frac{1}{V}\left(\frac{\partial V}{\partial P}\right)_{T, J, Q}
$$

(Non-rotating black holes are adiabatically incompressible, as fixing $S$ completely fixes $V$ when $J=0$.) This is one place where one can connect with astrophysical black holes as the compressibility (36) remains non-zero as $\Lambda \rightarrow 0$. The $Q=0$ black hole equation of state for a solar mass black hole is significantly stiffer than that of a neutron star of the same mass, assuming an ideal degenerate gas of neutrons.

An adiabatic speed of sound can be defined using the usual thermodynamic formula, defining an average density in a natural way $\rho=\frac{M}{V}$ a speed of sound, $v_{s}$, can be defined through

$$
v_{s}^{-2}=\left.\frac{\partial \rho}{\partial P}\right|_{S, J, Q}=1+\rho \kappa_{S}=1+\frac{9(2 \pi J)^{4}}{\left(6 S^{2}+16 P S^{3}+3(2 \pi J)^{2}\right)^{2}} \geq 1
$$

with equality for $J=0$, when the compressibility vanishes. Of course $v_{s}$ cannot be the speed of a surface wave on the black hole, it is probably better thought of as the velocity of a breathing mode due to the changing volume at constant $S$.

So far we have only discussed the properties of asymptotically AdS black holes with fixed angular momentum, which exhibit the CKK phase transition, and/or fixed charge, which exhibit the van der Waals type phase transition described in [50, 51. There is of course another kind of phase transition, first found by Hawking and Page [37, in which the free energy of the black hole with a given Hawking temperature is unstable and decays to AdS space-time, filled with radiation at the same temperature, due to competition between the free energies. The black hole will decay if its free energy is greater than that of AdS with no 
black holef and, conversely, if the Hawking temperature is such that the black hole as a lower free energy than AdS with radiation at the same temperature then black holes will be expected to spontaneously nucleate in AdS space-time. If the black hole has fixed non-zero charge or angular momentum then this is not an issue because conservation of charge/angular momentum stops any such transitions, but a rotating black hole with fixed angular velocity $\Omega$ can decay to AdS with rotating radiation at the same temperature (providing it is not rotating too fast - such a rotating black hole is asymptotically conformal to a 3-dimensional rotating Einstein universe and the angular velocity is limited if we do not want the Einstein universe to rotate faster than the speed of light [74]).

The free energy for a black hole at constant $\Omega$,

$$
\Xi(T, P, \Omega)=M-T S-\Omega J,
$$

is shown in figure 3 as a function of $T$, at fixed $P$. There are two branches, the upper branch is small black holes and the lower branch large black holes, the Hawking-Page phase transition occurs at the point where $\Xi$ becomes negative and large black holes are more stable than AdS with thermal radiation and no black hole, for which $\Xi=0$. There is a cusp in $\Xi$ at the point where the heat capacity diverges, but there is no second order phase transition as one cannot go through the cusp to lower $T$ - the black hole solution simply does not exist for lower $T$.

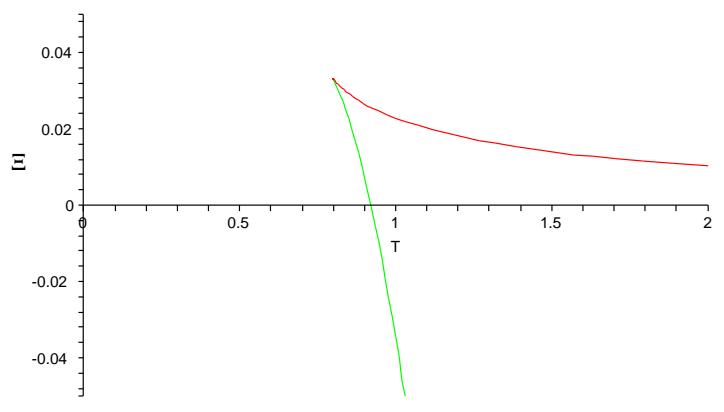

Figure 3: Free energy as a function of $T$ at fixed $P$ and $\Omega$. The point where the lower branch crosses the $T$-axis is the Hawking-Page phase transition.

Many other standard thermodynamic quantities can be checked in various situations:

- The Clapeyron equation for first order transitions, relating the slope of the phase boundary in the $P-T$ plane to the ratio of the jump in entropy to

\footnotetext{
${ }^{4}$ The individual free energies are infinite, but their difference is finite [37, alternatively [72, 73 the free energy can regularized by adding suitable counter terms at large $r$ and letting $r \rightarrow \infty$, both these procedures give the same answer.
} 
the jump in volume. For the Hawking-Page phase transition the jump in volume is $\Delta V=V$ (since $V=0$ in the AdS phase) and similarly $\Delta S=S$ and the Clapeyron equation reduces to

$$
\frac{d P}{d T}=\frac{\Delta S}{\Delta V}=\frac{S}{V} .
$$

For the Hawking-Page phase transition this evaluates to

$$
\frac{d P}{d T}=\frac{2 P S}{M},
$$

which is easily checked using the explicit solution. The latent heat across the first order transition is 75 .

$$
L=T \Delta S+\Omega \Delta J=\frac{16 \pi^{3} T^{3}}{\left(4 \pi^{2} T^{2}-\Omega^{2}\right)^{2}} .
$$

- For second order transitions $\Delta V=\Delta S=0$ and the Clapeyron equation is not directly applicable. The appropriate framework is then Ehrenfest's equations and these were investigated for charged, non-rotating black holes in [76- 78].

For rotating black holes Ehrenfest's equations are satisfied for neutral black holes at constant $\Omega,[79$ and also for charged black holes [80].

- The thermodynamic volume has also been explored in modified gravitational theories, beyond the simple Einstein action, such as: quantum corrected, asymptotically safe gravity [81; $f(R)$ gravity [82]; and conformal gravity [83].

- Quasi-normal mode frequencies show a marked change near second order phase transitions between large and small black holes [84, 85].

Another direction that has been pursued is to include the cosmological constant as a thermodynamical variable in the application of geometrothermodynamics to black holes [86]. Geometrothermodynamics investigates a natural geometry associated with the curvature of thermodynamic potentials (not to be confused with the geometry of space-time!). It was found in [87 that including $\Lambda$ as a thermodynamic variable dos not affect the singularity structure of the thermodynamic Ricci scalar.

\section{2 de Sitter black holes}

A positive cosmological constant has subtleties associated with it that are absent in the asymptotically AdS geometry: notably the presence of two horizons, a black hole and a cosmological horizon, each with different temperatures; the absence of a time-independent, space-like, asymptotic regime and resulting difficulties in defining a mass. Nevertheless progress can be made, using Komar 
forms to define an invariant mass 88 (resulting in essentially the same result as would be obtained by taking the AdS form of the mass 4 4 and analytically continuing to negative pressure). One outcome of this analysis is that, if the thermodynamic volume of the Universe is fixed, the entropy of the Universe is always increased if a black hole is added. The topic was further explored in [89, 90] and a position dependent mass function related to enthalpy proposed in 91, based on earlier work in 92.

Quintessence quintessentially involves a positive cosmological constant and the equation of state for Reissner-Nordström black holes in quintessence theories was shown in Ref. 93 again to have a large black hole/small black small black hole transition with a critical point possessing mean field exponents.

\subsection{Other $4-D$ space-times}

Following our general philosophy here one can ask questions about the volume associated to any space-time for which the Euclidean action, $I_{E}$, produces a non-trivial function depending on $P$. The Euclidean action is related to a thermodynamic potential (Massieu function 34) $\Xi(T, P, \Omega, \Phi)$, which is a function of purely intensive variables, via $\Xi=T I_{E}$ 94. For example asymptotically AdS Taub-Nut and Taub-bolt solutions were studied in Refs. 95- 97 while the Kerr-bolt was treated in Ref. 98. A curious feature is that the AdS-Taub-NUT thermodynamic volume is negative, a result that can be understood in terms a "formation process" of Taub-NUT space-time from pure AdS which has the effect of increasing the volume of space-time 95 .

\section{$4 D=3$ and the BTZ black hole}

Black holes in 3-dimensions have been a source of inspiration in gravitational research ever since the discovery of the BTZ solution [99, 100. They provide theoretical insights, both classical and quantum, into the physics of black holes which are much harder to analyse in 4-dimensions. For a review of BTZ black holes see [101.

The thermodynamic volume of non-rotating asymptotically AdS BTZ black holes was calculated in [17 and, as for other dimensions, it gives the Euclidean geometric result: in this case the area of a disc of radius $r_{h}, \pi r_{h}^{2}$. In three dimensions however one can make progress in including quantum gravity corrections to the free energy of the black hole 102. The thermodynamic volume arising from the quantum corrected free energy was calculated in [17 and it was found that the pressure was reduced at low volumes compared to the classical equation of state. This was the first example of a thermodynamic volume that differed from the naïve Euclidean geometry result. For the rotating BTZ there is no CKK critical point in 3-dimensions [45, a conclusion which extends to nonlinear Born-Infeld electrodynamics [103]. When scalar hair is included however a van der Waals type phase transition was reported in [104. Also when the Einstein action is modified to include a Chern-Simons term with torsion, as is 
very natural for the asymptotically AdS BTZ black hole, there are divergences in the heat capacity and the form of these divergences on the spinodal curve were investigated in [105.

\section{$5 \quad D>4$ space-time dimensions}

In more than 4-dimensions a black hole can have more than one angular momentum, due to the fact that the rank of the rotation group in $(D-1)$ space dimensions is greater than one and the number of independent angular momenta is equal to the rank $r$ of $S O(D-1)$, i.e. $\frac{D-2}{2}$ in even dimensions and $\frac{D-1}{2}$ in odd dimensions.

For charged static black holes in more than 4-dimensions the situation is similar to $4-D$, there is a critical point with mean field exponents. 45, 106, 107. The observation that the thermodynamic volume agrees with the naïve volume of a perfect sphere in Euclidean space also generalises to $D$ space-time dimensions [17, the thermodynamic volume evaluates to that of a $(D-2)$ dimensional sphere of radius $r_{h}$ in $(D-1)$ dimensional Euclidean space. As in $D=4$ this is no longer the case for rotating black holes 42 and the reverse isoperimetric inequality conjecture extends to all $D \geq 4$.

In $D>4$ the phase space structure of black holes rotating with constant angular momentum is much richer than in 4-dimensions. In addition there are black holes with event horizons which do not have the topology of a $D-2$ dimensional sphere, such as black rings [108] and black saturns [109, but the thermodynamic volume of such objects has yet to be investigated.

Even when all but one of the angular momenta are set to zero (singly spinning black holes) the structure of the thermodynamic phase space can be more involved than $D=4$. In all dimensions $D \geq 6$ singly spinning black holes can exhibit the phenomenon of re-entrant phase transitions where phases $A$ and $B$ alternate as $A-B-A$ when a single parameter is varied [110, in this case varying the temperature, at fixed pressure and angular momentum, with phase $A$ being large black holes and $B$ small black holes.

When more than one angular momentum is non-zero there are more possibilities. In $D=6$ the state of rotation is described by two angular momenta, $J_{1}$ and $J_{2}$, and the structure of the phase space depends on the ratio $J_{1} / J_{2}$. In addition to the CKK transition, familiar in 4-dimensions, there can now be multiple first-order transitions between three different phases: small, intermediate size and large black hole phases, as well as triple points [111, 112.

The adiabatic compressibility of higher dimensional asymptotically AdS rotating black holes, specifically asymptotically AdS Myers-Perry black holes, [13]-[116, was evaluated in [117]. It is positive and bounded above for $P>0$, but can diverge in the asymptotically flat limit $P \rightarrow 0$. The speed of sound associated with a Myers-Perry (asymptotically flat) black hole has an elegant expression in terms of quadratic and quartic Casimirs of $S O(D-1)$ when $\Lambda \rightarrow 0$ : defining dimensionless angular momenta as the angular momenta per unit entropy, $\mathcal{J}_{i}=\frac{2 \pi J_{i}}{S}$ with $i=1, \ldots, r$, the speed of sound, as $\Lambda \rightarrow 0^{-}$, evaluates 
to

$$
v_{s}^{2}=\frac{1}{(D-2)} \frac{\left(D-2+\sum_{i} \mathcal{J}_{i}^{2}\right)^{2}}{\left(D-2+2 \sum_{i} \mathcal{J}_{i}^{2}+\sum_{i} \mathcal{J}_{i}^{4}\right)},
$$

which lies in the range $\frac{1}{D-2}<v_{s}^{2} \leq 1$. Non-rotating black holes are incompressible, with $v_{s}^{2}=1$, while the lowest speed is given by $v_{s}^{2} \rightarrow \frac{1}{D-2}$, when on 5 or more $\mathcal{J}_{i} \rightarrow \infty$. This is still higher than the speed of sound in a photon gas in $D$-dimensions, which is $v^{2}=\frac{1}{D-1}$.

Local thermodynamic stability was discussed in 92 . The stability of a thermodynamic system depends on what external constraints are applied [39]. Including the cosmological constant as a thermodynamic variable raises questions about local thermodynamic stability over and above the usual considerations of positive heat capacity and positivity of the moment of inertia. The thermal energy $U\left(S, V, J_{i}, Q\right)$ is now a function of $r+3$ variables, where $r$ is the rank of $S O(D-1)$, and checking for convexity in all variables in complete generality requires examining the positivity properties of the eigenvalues of the $(r+3) \times(r+3)$ matrix $\partial_{A} \partial_{B} U$. When $P V$ is zero, or negative, this matrix always has a negative eigenvalue (see discussion around equation (18)) and the system cannot be stable. $P V>0$ is a necessary but not sufficient condition for local thermodynamic stability.

This question of local thermodynamic stability when $\Lambda$ is allowed to vary was addressed, for electrically neutral black holes, in [39. A sufficient set of conditions was given in terms of the adiabatic compressibility at constant angular momentum,

$$
\kappa_{J, S}=-\left.\frac{1}{V} \frac{\partial V}{\partial P}\right|_{J, S},
$$

the heat capacity at constant $P$ and $\Omega$,

$$
C_{\Omega, P}=\left.T \frac{\partial S}{\partial T}\right|_{\Omega, P}
$$

and the adiabatic momentum of inertia tensor,

$$
\mathcal{I}_{S, P}^{i j}=\left.\frac{\partial J^{i}}{\partial \Omega_{j}}\right|_{S, P}
$$

(this last is automatically symmetric since it is the inverse of $\left.\frac{\partial \Omega_{i}}{\partial J^{j}}\right|_{S, P}$ and $\Omega_{i}=$ $\left.\left.\frac{\partial H}{\partial J^{2}}\right|_{S, P}\right)$.

For electrically neutral asymptotically AdS Myers-Perry black holes there is indeed a completely stable region provided $P$ is large enough and the rotation co-efficients are not too large.

\footnotetext{
${ }^{5}$ Which can happen in $D \geq 5$, a phenomenon termed "ultra-spinning" black holes. This is associated with a divergence in the moment of momentum tensor, the angular velocity remains finite.
} 
The stability conditions can also be expressed in terms of constant $\Omega$ rather than constant $J$ : if everything is allowed to vary the question of complete local stability is independent of the variables used, though it may be expressed differently in different variables. For example in 4-dimensions, when $\Lambda=0, C_{J}$ is negative in some regions of parameter space and positive in others while the isothermal moment of inertia tensor, $\mathcal{I}_{T}$, has the opposite sign to $C_{J}$, but the product is always negative so there is no stable region. On the other hand $C_{\Omega}$ is always negative and $\mathcal{I}_{S}$ is always positive, so in $(S, \Omega)$ variables the instability is never visible in the moment of inertia. Local stability is of course a question of constraints and stability can be restored by fixing some variables. For example fixing $P$ and $J$ gives the CKK critical point in 4-dimensions, with a second order phase transition, while there is no such critical point at fixed $\Omega$.

The above discussion is in terms of local thermodynamic stability, determined by curvature properties of the thermodynamic potentials associated with specific solutions of Einsteins equations, but there is also the question of global stability: for a given set of the variables $(S, P, J, Q)$ is there another solution of Einstein's equations with a lower free energy $\Xi(T, P, \Omega, \Phi)$ ? The Hawking-Page phase transition is a consequence of the existence of such a solution. Fixing either $J$ or $Q$ eliminates the possibility of a Hawking-Page phase transition, because AdS without a black hole cannot have non-zero angular momentum or electric charge, whereas fixing $\Omega$ does not protect against Hawking-Page decay of black holes as AdS without a black hole can still rotate.

There is a number of possibilities for modified gravity and/or modified electromagnetism in $D>4$.

- Non-linear electrodynamics in general $D$, using the Einstein-Born-Infeld action, was treated in [45, 103, 118. For charged black holes there is a critical point in any $D \geq 4$, with mean field exponents [45]. The analytic form of the singularities in the heat capacity on the spinodal curve was determined in [118.

Another version of non-linear electrodynamics, known as the power Maxwell invariant form, which replaces $-F^{2}$ in the Maxwell action with $\left(-F^{2}\right)^{n}$ for some positive integer $n$, was considered in [119, 120, critical points with mean field exponents again emerged.

- The effect of varying the cosmological constant on the thermodynamics of Horava-Lifshitz black holes was investigated in [121.

- The $D$-dimensional Einstein-Maxwell action can be modified by adding a Gauss-Bonnet term. The Lagrangian becomes

$$
\mathcal{L}_{G-B}=\frac{1}{16 \pi G_{(D)}}\left\{R-2 \Lambda+a^{2}\left(R_{\mu \nu \rho \sigma} R^{\mu \nu \rho \sigma}-4 R_{\mu \nu} R^{\mu \nu}+R^{2}\right)\right\}-\frac{1}{4} F_{\mu \nu} F^{\mu \nu},
$$

where $G_{(D)}$ is Newton's constant in $D$-dimensions and $a$ is a constant with dimensions of length. For a charged black hole with a spherical event 
horizon there are critical points, associated with large black/small black hole phase transitions, with mean field exponents [122]-124.

For $D=5$ this can happen even when the black hole is electrically neutral but for $D \geq 6$ there must be a non-zero charge [122. In [125] it was found that, when the potential $\Phi$ is held fixed rather than the electric charge, the critical point persists only for $D=5$. The Ehrenfest equations for phase transitions associated with this action were checked in [107].

- Lovelock gravity [126] in $D$ dimensions is an extension of the Einstein action to include higher order terms in the Riemann tensor. It is the unique co-variant extension that gives equations of motion that involve at most second order derivatives of the metric. The Lagrangian for Lovelock gravity theory, including a cosmological constant, is given by

$$
\mathcal{L}=\frac{1}{16 \pi G_{(D)}}\left(R-2 \Lambda+\sum_{n=2}^{r} a_{n} \mathcal{L}_{n}\right)
$$

where $a_{n}$ are coupling constants, with dimensions of $(\text { length })^{2(n-1)}$, as before $r$ is the rank of $S O(D-1)$ and $\mathcal{L}_{n}$ are the $2 n$-dimensional Euler densities,

$$
\mathcal{L}_{n}=\frac{1}{2^{n}} \delta_{\left[\rho_{1} \sigma_{1} \ldots \rho_{n} \sigma_{n}\right]}^{\left[\mu_{1} \nu_{1} \ldots \mu_{n} \nu_{n}\right]} R_{\mu_{1} \nu_{1}}^{\rho_{1} \sigma_{1}} \ldots R_{\mu_{n} \nu_{n}}^{\rho_{n} \sigma_{n}},
$$

where $\delta_{\left[\rho_{1} \sigma_{1} \ldots \rho_{n} \sigma_{n}\right]}^{\left[\mu_{1} \nu_{1} \ldots \mu_{n} \nu_{n}\right]}$ represents products of $\delta$-functions totally antisymmetric in both sets of indices, (if only $a_{2}$ is non zero, Lovelock gravity reduces to Gauss-Bonnet gravity). The phase space is even further extended in these theories because in principle the $a_{n}$ can be considered to be varying thermodynamical variables as well as $\Lambda$. Charged black hole solutions for Lovelock gravity are known 127 and these were investigated, in the context of varying $\Lambda$ and extended phase space, for 3rd order Lovelock gravity in 128 - 130 . There are critical points in all dimensions $D \geq 7$, with some cases exhibiting multiple re-entrant phase transitions between small/large/small/large black holes as well as triple points. In [128, a critical point was found $D=7$, even for electrically neutral black holes.

For 3rd-order Lovelock theory in $D \geq 7$, with $a_{2}^{2}=3 a_{3}$, neutral black holes with event horizons that have hyperbolic geometry have non-trivial critical exponents, 130 $\alpha=0, \beta=1, \gamma=2$ and $\delta=3$. This is an example of non-mean field critical exponents, and the same exponents are also found in higher order Lovelock gravity [131].

3rd order Lovelock and Born-Infeld have also been considered in parallel [128, 132].

- A variation of Lovelock gravity, called quasi-topological gravity, was studied in 133 and the logic turned around to use the first law, with a $P d V$ term, to extract an expression for the entropy. 
- Other possibilities exist, such as including different kinds of matter in the action, 134.

The positive $\Lambda$ case has in $D>4$ has so far received very little attention, though charged black holes were shown to have phase transitions in [135.

In conclusion there is a wide range of higher order theories of gravity and/or non-linear electrodynamics in which first and second order phase transitions occur, in some cases an electric charge or rotation is not even necessary. Equations of state in the van der Waals class seem very robust, the only example of critical exponents that are not mean field known to date is associated with $r$-th order Lovelock gravity, with odd $r$ and a specific relation between the Lovelock co-efficients so that there only one extra independent parameter [131].

\section{Gauge/gravity duality}

When the cosmological constant is considered to be an independent thermodynamic variable it is very natural to ask what its thermodynamic interpretation might be in the boundary field theory of the AdS/CFT correspondence [136] (for a review see [137). In the best understood case a 10-dimensional supergravity solution, corresponding to $A d S_{5} \times S^{5}$, relates to $\mathcal{N}=4, S U(N)$ Yang-Mills theory on the 4-dimensional boundary of $A d S_{5}$, at large $N$. In this case the 5-dimensional $\Lambda$ of $A d S_{5}$ is related to the radius, $L$, of $S^{5}$ via

$$
\Lambda=-\frac{6}{L^{2}}
$$

and

$$
L^{4} \sim N \ell_{P}^{4}
$$

with $\ell_{P}$ the 1-dimensional Planck length. Putting a black hole in $A d S_{5}$ then allows finite temperature field theory to be studied on the boundary and the Hawking-Page phase transition in the bulk was identified with the de-confining phase transition in the boundary gauge theory in [138, 139.

It was first suggested in [5] that varying $\Lambda$ in the AdS/CFT picture should be equivalent to varying the number of colors, $N$, in the boundary Yang-Mills theory. This was also independently suggested in 444. The thermodynamic variable conjugate to $\Lambda$ would then act as a chemical potential, $\mu$, for color. This chemical potential was calculated, for $\mathcal{N}=4$ SUSY Yang-Mills at large $N$, in [140] and shown to have the general properties that a chemical would be expected to have, for example at large $T$ it is negative and is a decreasing function of $T$. For flat event horizons $\mu$ remains negative at small $T$ while for spherical event horizons it can pass through zero and become positive at a temperature that is only some $6 \%$ below the de-confining phase transition of the gauge theory proposed in [139].

In all of the above extensions of Einstein gravity and/or electrodynamics there are extra dimensionful parameters introduced into the action which affect scaling and the Smarr relation. In higher dimensions there can also be more 
dimensionful parameters in the solution, over and above the extra angular momenta associate with $r>1$. For example in planar black holes in $D$-dimensions one can associate different tensions in different tangential directions on the event horizon and the sum of the ADM mass and tensions was shown to vanish in 141, a result which is expected from the AdS/CFT correspondence.

The role of $\Lambda$ in the boundary field theory in 2-D dilaton gravity was explored in 142 .

\section{Conclusions}

When the cosmological constant was first introduced it was considered to be a fixed parameter, but over the years an increasing number of scenarios in which it might vary have been considered: for example it can be a parameter in a solution of higher dimensional gravity or it could be determined dynamically, as in inflationary models. In this context extending the thermodynamic phase space of black holes to include the cosmological constant as a thermodynamic variable seems a very natural move and it is perhaps somewhat surprising that it has taken so long for this step to be taken. The result is a much richer thermodynamics than heretofore, with second order phase transitions, triple points and re-entrant phase transitions. With the exception of higher order Lovelock gravity, all second order phase transitions studied so far have yielded classical mean field exponents, putting these gravitational systems in the same universality class as the van der Waals gas - indeed a solution of Einstein's equations corresponding to an energy momentum tensor with physically reasonable properties and giving exactly the van der Walls equation of state was constructed in 143. From a statistical mechanical point of view of course mean field behavior is to be expected in four or more dimensions, but until a bridge can be built between some microscopic quantum theory of quantum gravity and the thermodynamic potentials of black holes it is not clear how these exponents are related to any putative quantum gravity theory. Perhaps the deviation from mean field exponents found in some models of Lovelock gravity is a hint of quantum gravity effects being reproduced by the Lovelock action, but it is as yet too early to say. These ideas are still in the early stages of development and we can look forward to many more exciting developments in the future.

\section{References}

[1] R. Arnowitt, S. Deser and C.W. Misner, Phys. Rev. 117, (1960) 1595.

[2] R. Arnowitt, S. Deser and C.W. Misner, Phys. Rev. 118, (1960) 1100.

[3] R. Arnowitt, S. Deser and C.W. Misner, Phys. Rev. 122, (1961) 997.

[4] M. Henneaux and C. Teitelboim, Commun. Math. Phys. 98, (1985) 391. 
[5] D. Kastor, S. Ray and J. Traschen, Class. Quantum Grav. 26, (2009) 195011, arXiv:0904.2765.

[6] L. Smarr, Phys. Rev. Lett. 30, (1973) 71.

[7] M. Henneaux and C. Teitelboim, Phys. Lett. 143B, (1984) 415.

[8] M. Henneaux and C. Teitelboim, Phys. Lett. 222B, (1989) 195.

[9] C. Teitelboim, Phys. Lett. 158B, (1985) 293.

[10] Y. Sekiwa, Phys. Rev., D73, (2006) 084009, arXiv:hep-th/0602269.

[11] S. Wang, S-Q. Wu, F. Xie and L. Dan, Chin. Phys. Lett. 23, (2006) 1096, arXiv:hep-th/0601147.

[12] S. Wang, Thermodynamics of high dimensional Schwarzschild de Sitter spacetimes: variable cosmological constant, arXiv:gr-qc/0606109.

[13] W. Shuang, S-Q. Wu, X. Fei and D. Lin, Chin. Phys. Lett. 23, (2006) 1096, hep-th/0601147.

[14] A. Larrañaga, Stringy Generalization of the First Law of Thermodynamics for Rotating BTZ Black Hole with a Cosmological Constant as State Parameter, arXiv:0711.0012 [gr-qc]].

[15] M. Urano, A. Tomimatsu and H. Saida, Class. Quantum Grav. 26, (2009) 105010, arXiv:0903.4230 [gr-qc]].

[16] M-S. Ma and R. Zhao, Phys. Rev. D89, (2014) 044005, arXiv:1310.1491 [gr-qc]].

[17] B.P. Dolan, Class. Quantum Grav. 28, (2011) 125020, arXiv:1008.5023.

[18] S. A. Hayward, Class. Quantum Grav. 15, (1998) 3147, gr-qc/9710089.

[19] M. K. Parikh, Phys. Rev. D73, (2006) 124021, hep-th/0508108.

[20] W. Ballik and K. Lake, The volume of stationary black holes and the meaning of the surface gravity, arXiv:1005.1116 [gr-qc]].

[21] W. Ballik and K. Lake, Phys. Rev. D88, (2013) 104038, arXiv:1310.1935 [gr-qc]].

[22] R.M. Wald, General Relativity, (1984) Chicago University Press.

[23] S.W. Hawking, Nature 248, (1974) 30.

[24] S.W. Hawking, Comm. Math. Phys. 43, (1975) 199; erratum: ibid. 46, (1976) 206.

[25] S.W. Hawking, Phys. Rev. D13, (1976) 191. 
[26] J.D. Bekenstein, Lett. Nuovo. Cimento 4, (1972) 737.

[27] J.D. Bekenstein, Phys. Rev. D7, (1973) 2333.

[28] M.M. Caldarelli, C. Cognola and D. Klemm, Class. Quantum Grav. 17, (2000) 299, arXiv:hep-th/9908022.

[29] T. Padmanabhan, Class. Quantum Grav. 19, (2002) 5387, arXiv:gr-qc/0204019.

[30] J.T.G. Galvez Ghersi, G. Geshnizjani, F. Piazza and S. Shandera, JCAP 2011, (2011) 005, arXiv:1103.0783 [gr-qc]].

[31] J. W. Maluf, S. C. Ulhoa and J. F. da Rocha-Neto, Phys. Rev. D85, (2012) 044050, arXiv:1202.4995 [gr-qc]].

[32] S. C. Ulhoa and E. P. Spaniol, On Gravitational Entropy of de Sitter Universe, arXiv:1312.1367 [gr-qc]].

[33] K. H. C. Castello-Branco and J. F. da Rocha-Neto, Phys. Rev. D88, (2013) 2, 024045, arXiv:1312.7478 [gr-qc]].

[34] H.B. Callen, Thermodynamics and an Introduction to Thermostatistics, Wiley (2006).

[35] E.H. Lieb and J. Yngvason, The physics and mathematics of the second law of thermodynamics, Phys. Rep. 310, (1999) 1.

[36] G. W. Gibbons, M. J. Perry and C. N. Pope, Class. Quantum Grav. 22, (2005) 1503, hep-th/0408217.

[37] S.W. Hawking and D.N. Page, Comm. Math. Phys. 87, (1983) 577.

[38] O. J. C. Dias, P. Figueras, R. Monteiro, H. S. Reall and J. E. Santos, JHEP 1005, (2010) 076, arXiv:1001.4527 [hep-th]].

[39] B. P. Dolan, Thermodynamic stability of asymptotically anti-de Sitter rotating black holes in higher dimensions, Class. Quantum Grav. to appear, arXiv:1403.1507 [gr-qc]].

[40] A. Strominger and C. Vafa, Phys. Lett. 379B, (1996) 99, arXiv:hep-th/9601029.

[41] B. Carter, Comm. Math. Phys. 10, (1968) 280.

[42] M. Cvetic, G.W. Gibbons, D. Kubizn̆ák and C.N. Pope, Phys. Rev. D84, (2011) 024037, arXiv:1012.2888.

[43] B.P. Dolan, Class. Quantum Grav. 28, (2011) 235017, arXiv:1106.6260.

[44] C. V. Johnson, Holographic Heat Engines, arXiv:1404.5982 [hep-th]]. 
[45] S. Gunasekaran, R. B. Mann and D. Kubizn̆äk, JHEP 1211, (2012) 110, arXiv:1208.6251 [hep-th].

[46] B. P. Dolan, Where is the PdV term in the fist law of black hole thermodynamics?, in Open Questions in Cosmology (DOI:10.5772/52455) ed. G.J. Olmo (2012) InTech, arXiv:1209.1272 [gr-qc]].

[47] Y. D. Tsai, X. N. Wu and Y. Yang, Phys. Rev. D85 (2012) 044005, arXiv:1104.0502 [hep-th]].

[48] M. J. Sewell, Maximum and Minimum Principles (1990) Cambridge University Press, N.Y.

[49] V.I. Arnold, Dynamical systems (Encyclopedia of Mathematical Sciences, V), Chapter II, Catastrophe Theory (1994) Springer-Verlag.

[50] A. Chamblin, R. Emparan, C.V. Johnson and R.C. Myers, Phys. Rev. D60, (1999) 064018, hep-th/9902170.

[51] A. Chamblin, R. Emparan, C.V. Johnson and R.C. Myers, Phys. Rev. D60, (1999) 104026, hep-th/9904197.

[52] X.N. Wu, Phys. Rev. D62, (2000) 124023.

[53] A. Sahay, T. Sarkar and G. Sengupta, JHEP 1004, (2010) 118, arXiv:1002.2538 [hep-th]].

[54] A. Sahay, T. Sarkar and G. Sengupta, JHEP 1007, (2010) 082, arXiv:1004.1625 [hep-th]].

[55] C. Niu, Y. Tian and X. -N. Wu, Phys. Rev. D85, (2012) 024017, arXiv:1104.3066 [hep-th]].

[56] D. Kubizn̆äk and R. B. Mann, JHEP 1207, (2012) 033, arXiv:1205.0559 [hep-th]].

[57] A. Belhaj, M. Chabab, H. E. Moumni, L. Medari and M. B. Sedra, Chin. Phys. Lett. 30, (2013) 090402, arXiv:1307.7421.

[58] M. Born and L. Infeld, Proc. Roy. Soc. A144, (1934) 425.

[59] G.W. Gibbons, Rev. Mex. Fis. 49S1, (2003) 19, hep-th/0106059.

[60] S. Fernando and D. Krug, Gen. Rel. Grav. 35, (2003), hep-th/0306120.

[61] T.K. Dey, Phys. Lett. B595, (2004) 484, hep-th/0406169.

[62] R.-G. Cai, D.-W. Pang and A. Wang, Phys. Rev. D70, (2004) 124034, hep-th/0410158.

[63] R. Banerjee, S. Ghosh and D. Roychowdhury, Phys. Lett. 696B, (2011) 156, arXiv:1008.2644 [gr-qc]]. 
[64] A. Lala and D. Roychowdhury, Phys. Rev. D86, (2012) 084027, arXiv:1111.5991 [gr-qc]].

[65] R. Banerjee and D. Roychowdhury, Phys. Rev. D85, (2012) 044040, arXiv:1111.0147 [gr-qc]].

[66] S. Dutta, A. Jain and R. Soni, JHEP 1312, (2013) 60, arXiv:1310.1748.

[67] S.A. Hartnoll and P. Kovtun, Phys. Rev. D76, (2007) 066001, arXiv:0704.1160 [hep-th]].

[68] A. Bayntun, C.P. Burgess, B.P. Dolan and S-S. Lee, New J. Phys. 13, (2011) 035012, arXiv:1008.1917.

[69] E. Spallucci and A. Smailagic, Phys. Lett. 723B, (2013) 436, arXiv:1305.3379 [hep-th]].

[70] E. Spallucci and A. Smailagic, J. Grav. 2013, (2013) 525696, arXiv:1310.2186 [hep-th]].

[71] B. P. Dolan, Phys. Rev. D84, (2011) 127503, arXiv:1109.0198 [gr-qc]].

[72] M. Henningson and K. Skenderis, JHEP 9807, 023 (1998), arXiv:hep-th/9806087.

[73] V. Balasubramanian and P. Kraus, Comm. Math. Phys. 208, 413 (1999), arXiv:hep-th/9902121.

[74] S.W. Hawking, C.J. Hunter and M.M. Taylor-Robinson, Phys. Rev. D59, (1999) 064005, arXiv:hep-th/9811056.

[75] B.P. Dolan, Vacuum energy and the latent heat of black holes, arXiv:1407.4037.

[76] J-X. Mo and W-B. Liu, Phys. Lett. 727B, (2013) 336.

[77] Z. Zhao and J. Jing, Ehrenfest scheme for complex thermodynamic systems in full phase space, arXiv:1405.2640 [gr-qc]].

[78] J-X. Mo, G-Q. Li and W-B. Liu, Phys. Lett. 730B, (2014) 111.

[79] R. Banerjee S.K. Modak and S. Samanta, Phys. Rev. D84, (2011) 064024, arXiv:1005.4832.

[80] R. Banerjee, S.K. Modak and D. Roychowdhury, JHEP 1210, (2012) 125, arXiv:1106.3877.

[81] M-S. Ma and Y-Q. Ma, Critical behaviors of black hole in an asymptotically safe gravity with cosmological constant, arXiv:1405.7609 [hep-th]].

[82] J-X. Mo, Europhys. Lett. 105, (2014) 20003. 
[83] W. Xu and L. Zhao, Critical phenomena of static charged AdS black holes in conformal gravity, arXiv:1405.7665 [gr-qc]].

[84] R. Tharanath, N. Varghese and V. C. Kuriakose, Mod. Phys. Lett. A29, (2014) 1450057, arXiv:1404.0791 [gr-qc]].

[85] Y. Liu, D-C. Zou and B. Wang, Signature of the Van der Waals like small-large charged AdS black hole phase transition in quasinormal modes, arXiv:1405.2644 [hep-th]].

[86] G. Ruppeiner, Thermodynamic curvature and black holes, arXiv:1309.0901.

[87] A. Larrañaga and S. Mojica, Abraham Zelmanov Journal 5, (2012) 68, arXiv:1204.3696 [gr-qc]].

[88] B. P. Dolan, D. Kastor, D. Kubizn̆äk, R. B. Mann and J. Traschen, Phys. Rev. D87, (2013) 10, 104017, arXiv:1301.5926 [hep-th]].

[89] M-S. Ma, H-H. Zhao, L-C. Zhang and R. Zhao, Int. J. Mod. Phys. A29, (2014) 1450050, arXiv:1312.0731 [hep-th]].

[90] R. Zhao, M. Ma, H. Zhao and L. Zhang Adv. in High Energy Physics 2014, (2014) 124854.

[91] S. Bhattacharya and A. Lahiri, Eur. Phys. J. C 73, (2013) 2673, arXiv:1301.4532 [gr-qc]].

[92] L.F. Abbott and S. Deser, Nucl. Phys. B195, (1982) 76.

[93] G. -Q. Li, Phys. Lett. 735B, (2014) 256, arXiv:1407.0011 [gr-qc]].

[94] G.W. Gibbons, M.J. Perry and C.N. Pope, Class. Quantum Grav. 22, (2005) 1503, hep-th/0408217.

[95] C. V. Johnson, Quantum Gravity and the Nuts and Bolts of Thermodynamic Volumes, arXiv:1405.5941 [hep-th]].

[96] C.V. Johnson, The Extended Thermodynamic Phase Structure of TaubNUT and Taub-Bolt, arXiv:1406.4533.

[97] C. O. Lee, The Extended Thermodynamic Properties of Taub-NUT/BoltAdS spaces, arXiv:1408.2073 [hep-th]].

[98] S. MacDonald, Thermodynamic Volume of Kerr-bolt-AdS Spacetime, arXiv:1406.1257 [hep-th]].

[99] M. Banados, C. Teitelboim and J. Zanelli, Phys. Rev. Lett., 69, (1992) 1849.

[100] M. Banados, C. Teitelboim and J. Zanelli, Phys. Rev., D48, (1993) 1506. 
[101] S. Carlip, Class. Quantum Grav. 12, (1995) 2853, arXiv:gr-qc/9506079.

[102] A. Maloney and E. Witten, JHEP 1002, (2010) 029, arXiv:0712.0155[hep-th]].

[103] D-C. Zou, S-J. Zhang and B. Wang, Phys. Rev. D89, (2014) 044002, arXiv:1311.7299 [hep-th]].

[104] A. Belhaj, M. Chabab, H. El Moumni and M. B. Sedra Critical Behaviors of 3D Black Holes with a Scalar Hair, arXiv:1306.2518 [hep-th]].

[105] M-S. Ma, F. Liu and R. Zhao, Class. Quantum Grav. 31, (2014) 095001, arXiv:1403.0449 [gr-qc]].

[106] A. Belhaj, M. Chabab, H. El Moumni and M. B. Sedra, Chin. Phys. Lett. 29, (2012) 100401, arXiv:1210.4617 [hep-th]].

[107] J-X. Mo and W-B. Liu, Phys. Rev. D89, (2014) 084057, arXiv:1404.3872 [gr-qc]].

[108] R. Emparan and H. S. Reall, Phys. Rev. Lett. 88, (2002) 101101, hep-th/0110260.

[109] H. Elvang and P. Figueras, JHEP 0705, (2007) 050, hep-th/0701035.

[110] N. Altamirano, D. Kubizn̆äk and R.B. Mann, Phys. Rev. D88, (2013) 101502, arXiv:1306.5756.

[111] N. Altamirano, D. Kubiznäk, R. B. Mann and Z. Sherkatghanad, Galaxies 2, (2014) 89, arXiv:1401.2586 [hep-th]].

[112] N. Altamirano, D. Kubizn̆ák, R. B. Mann and Z. Sherkatghanad, Class. Quantum Grav. 31, (2014) 042001, arXiv:1308.2672 [hep-th]].

[113] R.C. Myers and M.J. Perry, Annals Phys. 172, (1986) 304.

[114] S.W. Hawking, C.J. Hunter and M.M. Taylor-Robinson, Phys. Rev. D59, (1999) 064005, hep-th/9811056.

[115] G.W. Gibbons, H. Lü, D.N. Page and C.N. Pope, Phys. Rev. Lett. 93, (2004) 171102, hep-th/0409155.

[116] G.W. Gibbons, H. Lü, D.N. Page and C.N. Pope, Geom. Phys. 53, (2005) 49, hep-th/0404008.

[117] B. P. Dolan, Class. Quantum Grav. 31, (2014) 035022, arXiv:1308.5403 [gr-qc]].

[118] R. Banerjee and D. Roychowdhury, Phys. Rev. D85, (2012) 104043, arXiv:1203.0118 [gr-qc]]. 
[119] S. H. Hendi and M. H. Vahidinia, Phys. Rev.D88, (2013) 084045, arXiv:1212.6128 [hep-th]].

[120] G. Arciniega and A. Sánchez, Geometric description of the thermodynamics of a black hole with power Maxwell invariant source, arXiv:1404.6319 [math-ph]].

[121] J. Sadeghi, K. Jafarzade and B. Pourhassan, Int. J. Theor. Phys. 51, (2012) 3891.

[122] W. Xu, H. Xu and L. Zhao, Gauss-Bonnet coupling constant as a free thermodynamical variable and the associated criticality, arXiv:1311.3053 [grqc]].

[123] R-G. Cai, L-M. Cao, L. Li and R-Q. Yang, JHEP 1309, (2013) 005, arXiv:1306.6233 [gr-qc]].

[124] S-W. Wei and Y-X. Liu, Triple points and phase diagrams in the extended phase space of charged Gauss-Bonnet black holes in AdS space, arXiv:1402.2837 [hep-th]].

[125] D-C. Zou, Y. Liu and B. Wang, Critical behavior of charged Gauss-Bonnet AdS black holes in the grand canonical ensemble, arXiv:1404.5194 [hep-th]].

[126] D. Lovelock, J. Math. Phys. 12, (1971) 498.

[127] C. Charmousis, Lect. Notes Phys. 769, (2009) 299, arXiv:0805.0568 [grqc]].

[128] J-X. Mo and W-B. Liu, Eur. Phys. J. C74, (2014) 2836, arXiv:1401.0785 [gr-qc]].

[129] H. Xu, W. Xu and L. Zhao, Extended phase space thermodynamics for third order Lovelock black holes in diverse dimensions, arXiv:1405.4143 [grqc]].

[130] A. M. Frassino, D. Kubiznak, R. B. Mann and F. Simovic, Multiple Reentrant Phase Transitions and Triple Points in Lovelock Thermodynamics, arXiv:1406.7015 hep-th].

[131] B.P. Dolan, A. Kostouki, D. Kubizn̆äk and R.B. Mann, Isolated critical point from Lovelock gravity, arXiv:1407.4783.

[132] A. Belhaj, M. Chabab, H. E. Moumni, K. Masmar and M. B. Sedra, Ehrenfest Scheme of Higher Dimensional Topological AdS Black Holes in Lovelock-Born-Infeld Gravity, arXiv:1405.3306 [hep-th]].

[133] A. Sheykhi, M. H. Dehghani and R. Dehghani, Gen. Rel. Grav. 46, (2014) 1679, arXiv:1404.0260 [gr-qc]]. 
[134] E. Spallucci and A. Smailagic, Int. J. Mod. Phys. D22, (2013) 1350010, arXiv:1212.5044.

[135] L-C. Zhang, M-S. Ma, H-H. Zhao and R. Zhao, Thermodynamics of phase transition in higher dimensional Reissner-Nordström-de Sitter black hole, arXiv:1403.2151.

[136] J. Maldacena, Adv. Theor. Math. Phys. 2, (1998) 252, [arXiv:9711200].

[137] O. Aharony, S. S. Gubser, J. M. Maldacena, H. Ooguri and Y. Oz, Phys. Rept. 323, (2000) 183, hep-th/9905111.

[138] E. Witten, Adv. Theor. Math. Phys. 2, (1998) 253, arXiv:hep-th/9802150.

[139] E. Witten, Adv. Theor. Math. Phys. 2, (1998) 505, arXiv:hep-th/9803131.

[140] B. P. Dolan, Bose condensation and branes, arXiv:1406.7267.

[141] B. Mahmoud El-Menoufi, B. Ett, D. Kastor and J. Traschen, Class. Quantum Grav. 30, (2013) 155003, arXiv:1302.6980 [hep-th]].

[142] D. Grumiller, R. McNees and J. Salzer, Cosmological constant as confining $U(1)$ charge in two-dimensional dilaton gravity, arXiv:1406.7007.

[143] A. Rajagopal, D. Kubiznak and R. B. Mann, Van der Waals black hole, arXiv:1408.1105 [gr-qc]]. 